\title{
ATORMAC- $C_{V}$ ATORMAC GOLD \# Nurogab NT
}

NEUROLOGY INDIA

Open access journal indexed with Index Medicus

\begin{tabular}{|c|c|c|c|c|c|c|c|c|c|}
\hline$\underline{\text { About }}$ & $\begin{array}{l}\text { Current } \\
\text { Issue }\end{array}$ & $\underline{\text { Archive }}$ & $\frac{\text { Ahead of }}{\text { print }}$ & $\underline{\text { Search }}$ & Instructions & $\begin{array}{c}\text { Online } \\
\text { Submission }\end{array}$ & Subscribe & $\underline{\text { Etcetera }}$ & Contact \\
\hline
\end{tabular}

Navigate Here

Impact Factor for 2016

Search

Resource Links

» Similar in PUBMED

» Search Pubmed for

- e Sá MC

\section{" Search in Google} Scholar for

- e Sá MC

» Article in PDF $(410 \mathrm{~KB})$

" Citation Manager

" Access Statistics

" Reader Comments

" Email Alert *

" Add to My List *

* Registration required

(free)

\section{In this Article}

» References

\section{Article Access Statistics}

Viewed

Printed

Emailed

PDF Downloaded

13

Comments

[Add]

\section{Previous Article ToC Next Article}

\section{NI FEATURE: THE EDITORIAL DEBATE III-- PROS AND CONS}

Year : 2018 | Volume : 66 | Issue : 1 | Page : 51-52

The case for using lumbar drainage intraoperatively to help curb the number of iatrogenic CSF leaks

Manuel Cunha e Sá

Department of Neurosurgery, Hospital Garcia de Orta, Almada; Department of Neurosurgery and Neurosciences, Hospital Cuf/Infante Santo/Tejo, Lisboa,

Portugal

$\begin{array}{ll}\text { Date of Web } & \text { 11-Jan- } \\ \text { Publication } & 2018\end{array}$

\section{Correspondence Address:}

Dr. Manuel Cunha e Sá

Department of Neurosurgery, Hospital Garcia de Orta, Almada

Portugal

12. Login to access the email ID

Source of Support: None, Conflict of Interest: None

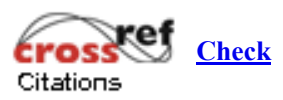

DOI: $10.4103 / 0028-3886.222842$

Get Permissions

for commercial use
How to cite this article:

e Sá MC. The case for using lumbar drainage intraoperatively to help curb the number of iatrogenic CSF leaks. Neurol India 2018;66:51-2

How to cite this URL:

e Sá MC. The case for using lumbar drainage intraoperatively to help curb the number of iatrogenic CSF leaks. Neurol India [serial online] 2018 [cited 2018 


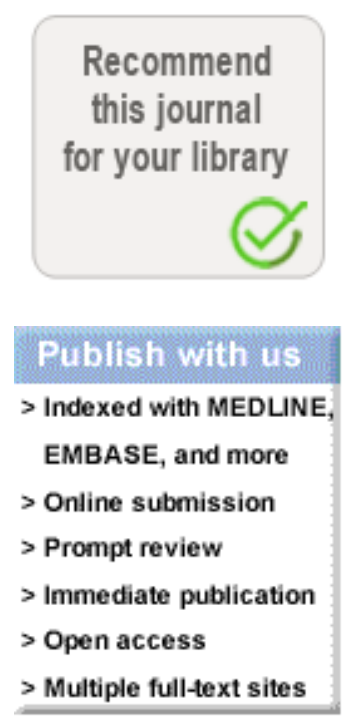

Click on image for details.
Feb 4];66:51-2. Available from: http://www.neurologyindia.com/text.asp? $\underline{2018 / 66 / 1 / 51 / 222842}$

Jonathan et al., have given themselves the task of analyzing their own surgical series of patients consecutively operated on for the resection of pituitary adenomas, randomizing them into two arms, one with the use of intraoperative lumbar drainage and the other without it. $[1]$

As stated by the authors and quoting their text, the primary outcome was the incidence of intraoperative cerebrospinal fluid (CSF) leak determined by endoscopic inspection at the end of surgery. Secondary outcome measures included the occurrence of postoperative CSF leaks and the extent of tumor resection using volumetric analysis on magnetic resonance imaging (MRI).

Sixty patients were systematically accrued for the study. All patients had a preoperative gadolinium enhanced MRI study performed but only 30 patients were said to be eligible for a postoperative scan. Moreover, these studies were not done under similar conditions with a limited number of patients undergoing postoperative gadolinium-enhanced studies. It is not clear how many patients underwent what type of study in each of the arms. The authors base their choice for limiting postoperative contrast studies on seemingly logical reasons.

Gadolinium was only administered in nonfunctional tumors, or in functional lesions with evidence of persisting endocrine disease. The argument is acceptable but is, nonetheless, a drawback of the study. In a randomized study like the current one, it would have been more appropriate to screen all patients in the same manner and search for the conclusions afterwards. The cohort of patients studied in each arm is limited, which makes it more difficult to dilute the effect of any possible single case that may fall out of the expected estimates.

All patients were said to have been advised to undergo a 3-month follow up but neither the total number of patients who actually did so, nor the number of patients who underwent a postoperative contrast MRI amongst them, have been stated.

The fact that patients with functional tumors are in remission at a three-month follow-up period is certainly a good indicator of the efficacy of surgical treatment administered but it is not an absolute one. In order to really assess the extent of the resection and, therefore, the likelihood of persisting remission/cure, one has to follow up these patients further up in time. In cases where the remission persists, I agree with the authors that it is licit to admit that the resection of the functional part of the tumor was complete. However, in the cases where reactivation of endocrinal disease is documented, these patients will need to be re-screened from the imaging standpoint, at which stage the lack of a comparative postoperative contrast enhanced MRI will become a problem.

Following the same line of thought, one can argue that the fact that only patients with nonfunctional tumors, or those with a functional lesion with a large suprasellar component were volumetrically screened in the immediate postoperative period, is also a methodological problem. The criteria for choosing who goes for what type of screening (immediate postoperative or at a three month follow-up) is unclear due to a non-homogeneous analysis. One could eventually accept the criteria of size (the large suprasellar component). It is, however, more difficult to indulge with the choice of applying the criteria of only the size of the tumor to functional tumors (where even small-sized tumors may have become symptomatic), or in the same manner to nonfunctional tumors (where the tumors may have resulted in clinical symptoms regardless of their size). 
Again, all these confounding factors create too much noise for a series of this size, which may importantly limit the conclusions of the study as far as the degree of resection goes.

In what relates to the primary endpoint, which is the existence of an intraoperative CSF fistula, we have to concurr that the difference is substantial between the two arms, so that the use of an intraoperative lumbar drain seems to work as a positive adjunct in helping the surgeon avoid violating the subarachnoid lining.

The authors state that their increasing experience in dealing with the resection of these tumors both with the microscopic, and currently, with the endoscopic technique has enabled them to curb the number of possible intraoperative leaks. This is true for all surgeons. One could also question why the option of an extracapsular dissection was not selectively applied for some tumors where this plan was well-defined. How does or would the intraoperative CSF drainage influence this type of dissection where the 'push and pull' technique of tumor delivery is also applied?

We are not given a reason why in the presence of a verified intraoperative CSF leak in patients in the group where a lumbar drainage was not initially undertaken, the therapeutic lumbar drainage was placed not in the operating room but within the next six hours in the intensive care unit.

The discussion conveniently covers most aspects related to the purported benefits of the use of an intraoperative lumbar drainage for the resection of pituitary tumors, which is mostly based on the more recent series of Mehta and Oldfield. [2] Similarly, arguments related to the possible complications of lumbar drainage are addressed in the text.

The case for the occurrence of spontaneous intracerebral hemorrhage associated with the use of lumbar drainage should also be highlighted despite its low incidence. In fact, many of the complications occurring with the use of lumbar drainage are to be found in the cardiothoracic literature. [3],[4]

Lumbar drainage is a procedure which involves a low risk for the patient. Rarely, it may carry deleterious consequences with transient but also permanent neurological damage. This argument should advise us to make a selected and appropriate use of this therapeutic weapon, gauging carefully the caliber of the Touhy needle and the catheter used and the amount of CSF drained.

At a first glance, the fact that we may be adding up morbidity to the index procedure by using an intraoperative lumbar drainage may curb our intention to use it altogether. This is not justifiable only on these terms if for no other reason other than the fact that one of the primary therapeutic attitudes recommended for the closure of a postoperative CSF fistula in the aftermath of pituitary surgery is... the placement of a continuous lumbar drainage system.

In the end, it is fair to say that the use of an intraoperative CSF drainage may be a helpful tool in reducing the percentage of iatrogenic CSF leaks. The experience of the authors, reinforced by similar reports from other senior surgeons in the field, [4] should capture our attention to this technical note.

$»$ References

1. Jonathan GE, Sarkar S, Singh G, Mani S, Thomas R, Chacko AG. A randomized controlled trial to determine the role of intraoperative lumbar 
cerebrospinal fluid drainage in patients undergoing endoscopic

transsphenoidal surgery for pituitary adenomas. Neurol India 2018;66:133-8. $\uparrow$

[Full text]

2. Mehta GU, Oldfield EH. Prevention of intraoperative cerebrospinal fluid leaks by lumbar cerebrospinal fluid drainage during surgery for pituitary macroadenomas. Journal of Neurosurgery, 2012;116:1299-303.

3. Wynn M, Mell M, Tefera G, Hoch JR, Acher C. Complications of spinal fluid drainage in thoracoabdominal aortic aneurysm repair: A report of 486 patients treated from 1987 to 2008. Journal of Vascular Surgery, January 2009;29-35. $\uparrow$

4. Fedorow C, Moon M, Mutch A, Grocott H. Lumbar cerebrospinal fluid drainage for thoracoabdominal aortic surgery: Rationale and practical considerations for management. Anesthesia Analgesia, July 2010, vol. 111, number $1,48-58$.

$\underline{\text { Site Map }}|\underline{\text { Home }}| \underline{\text { Contact Us }}|\underline{\text { Advertise With Us }}| \underline{\text { Feedback }} \mid \underline{\text { Copyright and Disclaimer }}$

Online since $20^{\text {th }}$ March '04

Published by Wolters Kluwer - $\underline{\text { Medknow }}$

Editorial and Ethics Policies

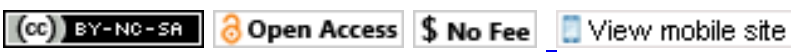

ISSN: Print -0028-3886, Online - 1998-4022 